\title{
Lattice Fuzzy Signal Operators and Generalized Image Gradients
}

\author{
Petros Maragos, Vassilis Tzouvaras, and Giorgos Stamou \\ National Technical University of Athens, \\ School of Electrical \& Computer Engineering, \\ Zografou, Athens 15773, Greece. \\ Email: maragos@cs.ntua.gr, tzouvaras@image.ntua.gr, \\ gstam@softlab.ntua.gr
}

\begin{abstract}
In this paper we use concepts from the lattice-based theory of morphological operators and fuzzy sets to develop generalized lattice image operators that are nonlinear convolutions that can be expressed as supremum (resp. infimum) of fuzzy intersection (resp. union) norms. Our emphasis and differences with many previous works is the construction of pairs of fuzzy dilation (sup of fuzzy intersection) and erosion (inf of fuzzy implication) operators that form lattice adjunctions. This guarantees that their composition will be a valid algebraic opening or closing. We have experimented with applying these fuzzy operators to various nonlinear filtering and image analysis tasks, attempting to understand the effect that the type of fuzzy norm and the shape-size of structuring function have on the resulting new image operators. We also present some theoretical and experimental results on using the lattice fuzzy operators, in combination with morphological systems or by themselves, to develop some new edge detection gradients which show improved performance in noise.
\end{abstract}

\section{Introduction}

Mathematical morphology (MM) and fuzzy sets share many common theoretical concepts. As an earlier example, the use of $\mathrm{min} / \mathrm{max}$ to extend the intersection/union of ordinary (crisp) sets to fuzzy sets [14] has also been used to extend the set-theoretic morphological shrink/expand operations on binary images to $\mathrm{min} /$ max filtering on graylevel images [11,3]. While the field of morphological image analysis was maturing, several researchers developed various other approaches using fuzzy logic ideas for extending or generalizing the morphological image operations $[13,1]$. The main ingredients of these approaches have been to (1) map the max-plus structure of Minkowski signal dilation to a sup- $T$ signal convolution, where $T$ is some fuzzy intersection norm, and (2) use duality to map the inf-minus structure of Minkowski signal erosion to a inf- $T^{\prime}$ convolution, where $T^{\prime}$ is a dual fuzzy union norm. The main disadvantage of these approaches is that composition of the operators from steps (1) and (2) is not guaranteed to be an algebraic opening or closing. (Openings and closing are the basic morphological smoothing filters.) 
Meanwhile MM was extended using lattice theory $[12,4]$ to more general operators that shared with the standard dilation, erosion, opening and closing only a few algebraic properties. One such fundamental algebraic structure is a pair of erosion/dilation operators that form an adjunction. This guarantees the formation of openings and closings.

In a previous work $[7,8]$ some of us used lattice theory to develop generalizations of morphological signal and vector operations based on fuzzy norms. These operations were used in fuzzy dynamical systems to represent the mapping between input and ouput signals (via nonlinear fuzzy-based convolutions) and the mapping between state vectors (via generalized fuzzy-based products of matrices and vectors) as a generalized dilation or erosion acting on signal or vector lattices. In this paper, which is a sequel of [9], we continue this work and apply our general theoretical results from [7] to developing useful nonlinear operators for image/signal analysis based on lattices and fuzzy set operations. From fuzzy set theory [6] we use t-norms and t-conorms to extend intersection and union of crisp sets to signal convolutions. To form openings and closings we use pairs of t-norms and fuzzy implications. (A work similar to our theoretical analysis appeared recently in [2]. Also, some recent work in fuzzy MM includes [5, 10].) First, we discuss the theoretical development of the new operators. We also present some results on using the lattice fuzzy operators, in combination with morphological systems or by themselves, to develop some new edge detection gradients which show improved performance in noise. Proofs of our theoretical results will be given in a longer paper.

\section{Background: Lattice Morphological Operators}

A poset $\mathcal{L}$ is any set equipped with a partial ordering $\leq$. The supremum $(\bigvee)$ and infimum $(\bigwedge)$ of any subset of $\mathcal{L}$ is its lowest upper bound and greatest lower bound, respectively, induced by the partial order; both are unique if they exist. The algebra $(\mathcal{L}, \vee, \wedge)$ is called a complete lattice if the supremum and infimum of any (finite or infinite) collection of its elements exists. An operator $\psi$ on a complete lattice $\mathcal{L}$ is called: increasing if it preserves the partial ordering $[f \leq g \Longrightarrow \psi(f) \leq \psi(g)]$; idempotent if $\psi^{2}=\psi$; antiextensive (resp. extensive) if $\psi(f) \leq f$ (resp. $f \leq \psi(f))$. An operator $\varepsilon$ (resp. $\delta$ ) on a complete lattice is called an erosion (resp. dilation) if it distributes over the infimum (resp. supremum) of any collection of lattice elements; namely $\delta\left(\bigvee_{i} f_{i}\right)=\bigvee_{i} \delta\left(f_{i}\right)$ and $\varepsilon\left(\bigwedge_{i} f_{i}\right)=\bigwedge_{i} \varepsilon\left(f_{i}\right)$. An operator is called an opening (resp. closing) if it is increasing, antiextensive (resp. extensive) and idempotent. An operator pair $(\varepsilon, \delta)$ is called an adjunction iff $\delta(f) \leq g \Longleftrightarrow f \leq \varepsilon(g), \forall f, g \in \mathcal{L}$. Given a dilation $\delta$, there is a unique erosion $\varepsilon(g)=\bigvee\{f: \delta(f) \leq g\}$ such that $(\varepsilon, \delta)$ is adjunction, and vice-versa.

Proposition 1 ([12], [4]). Let $(\varepsilon, \delta)$ be an adjunction. Then: (i) $\delta$ is a dilation and $\varepsilon$ is an erosion. (ii) $\delta \varepsilon$ is an opening, and $\varepsilon \delta$ is a closing.

For lattice-based image/signal processing, the signal space is the collection $\mathcal{L}=\mathbb{V}^{\mathbb{E}}$ of all images/signals $f: \mathbb{E} \rightarrow \mathbb{V}$, (a continuous or discrete) domain

Proc. 10th International Fuzzy Systems Association World Congress (IFSA03), Istanbul, Turkey, July 2003. Lecture Notes in Computer Science 2715, Springer 2003, pp.412-419. 
$\mathbb{E}$ where $\mathbb{E}=\mathbb{R}^{m}$ or $\mathbb{Z}^{m}, m=1,2, \ldots$, , and assuming values in $\mathbb{V} \subseteq \overline{\mathbb{R}}$ where $\overline{\mathbb{R}}=\mathbb{R} \cup\{-\infty, \infty\}$. The value set $\mathbb{V}$ must be a complete lattice under the usual ordering $\leq$ of real numbers, with corresponding sup $(\vee)$ and inf $(\wedge)$ the usual supremum and infimum in $\overline{\mathbb{R}}$. The signal space $\mathcal{L}$ also becomes a complete distributive lattice if we define on it the standard pointwise partial ordering $\leq$, supremum $\vee$, and infimum $\wedge$ induced by $\mathbb{V}$.

Proposition $2([4])$. The pair $(\varepsilon, \delta)$ is an adjunction on the signal lattice $\mathbb{V}^{\mathbb{E}}$ iff for every $x, y \in \mathbb{E}$ there exists a scalar adjunction $\left(\varepsilon_{y, x}, \delta_{x, y}\right)$ on $\mathbb{V}$ such that

$$
\delta(f)(x)=\bigvee_{y \in \mathbb{E}} \delta_{x, y}(f(y)), \quad \varepsilon(g)(y)=\bigwedge_{x \in \mathbb{E}} \varepsilon_{y, x}(g(x))
$$

\section{$3 \quad$ Lattice Operators using Fuzzy Norms}

In this paper we shall work on the complete signal lattice $\mathcal{L}=\mathbb{V}^{\mathbb{E}}$ where the range of all signals is the complete scalar lattice $\mathbb{V}=[0,1]$.

The classic translation-invariant (TI) dilations and erosions on $\overline{\mathbb{R}}^{\mathbb{E}}$ are built as sup of signal translations of the type $\tau_{y, v}(f)(x)=v+f(x-y)$. In this paper we shall use new translations where the binary operation $a+b$ will be replaced by fuzzy norms. Specifically, we build generalized TI image dilations and erosions by defining the scalar dilations (erosions) $\delta_{x, y}\left(\varepsilon_{y, x}\right)$ of (1) via some fuzzy intersection (union) between the values of the image signal $f$ and a structuring function $h$. First we define fuzzy norms.

A fuzzy intersection norm, in short a Tnorm, is a binary operation $T$ : $[0,1]^{2} \rightarrow[0,1]$ that satisfies the following conditions [6]: For all $a, b, c \in[0,1]$

F1. $T(a, 1)=a$ and $T(a, 0)=0$ (boundary conditions).

F2. $T(a, T(b, c))=T(T(a, b), c)$ (associativity).

F3. $T(a, b)=T(b, a)$ (commutativity).

F4. $b \leq c \Longrightarrow T(a, b) \leq T(a, c)$ (increasing).

For the Tnorm to be a scalar dilation (with respect to any argument) on $\mathbb{V}$, it must also satisfy [7]:

F5. $T$ is a continuous function.

A fuzzy union norm $[6]$ is a binary operation $U:[0,1]^{2} \rightarrow[0,1]$ that satisfies F2-F5 and a dual boundary condition:

$\mathrm{F} 1^{\prime} . U(a, 0)=a$ and $U(a, 1)=1$.

Clearly, $U$ is an erosion on $\mathbb{V}$.

Now the new signal translations on $\mathcal{L}=[0,1]^{\mathbb{E}}$ are the operators $\tau_{y, v}(f)(x)=$ $T(v, f(x-y))$, where $(y, v) \in \mathbb{E} \times \mathbb{V}$ and $f(x)$ is an arbitrary input signal. A signal operator on is called translation invariant (TI) iff it commutes with any such translation. Similarly, we define dual signal translations $\tau_{y, v}^{\prime}(f)(x)=$ $U(v, f(x-y))$. Consider now two elementary signals, called the impulse $q$ and the dual impulse $q^{\prime}$ :

$$
q(x) \triangleq\left\{\begin{array}{l}
1, x=0 \\
0, x \neq 0
\end{array}, \quad q^{\prime}(x) \triangleq\left\{\begin{array}{l}
0, x=0 \\
1, x \neq 0
\end{array}\right.\right.
$$

Proc. 10th International Fuzzy Systems Association World Congress (IFSA03), Istanbul, Turkey, July 2003. Lecture Notes in Computer Science 2715, Springer 2003, pp.412-419. 
Then every signal $f$ can be represented as a sup of translated impulses or as inf of dual-translated dual impulses:

$$
f(x)=\bigvee_{y} T[f(y), q(x-y)]=\bigwedge_{y} U\left[f(y), q^{\prime}(x-y)\right]
$$

General TI signal dilation and erosion can result, respectively, from the sup$T$ convolution $\bigcirc_{T}$ and the inf $-U$ convolution $\bigcirc_{U}^{\prime}$ of two signals $f$ and $g$ defined by

$$
\left(f \bigcirc_{T} g\right)(x) \triangleq \bigvee_{y} T[f(y), g(x-y)], \quad\left(f \bigcirc_{U}^{\prime} g\right)(x) \triangleq \bigwedge_{y} U[f(y), g(x-y)]
$$

The following theorem characterizes all TI signal dilation or erosion operators as nonlinear convolutions of the above type.

Theorem 1. ([7]). An operator $\Delta$ (resp. $\mathcal{E})$ on the signal lattice $[0,1]^{\mathbb{E}}$ is a translation invariant dilation (resp. erosion) iff it can be represented as the sup$T$ (resp. inf-U) convolution of the input signal with the operator's (resp. dual) impulse response $h=\Delta(q)$ [resp. $\left.h^{\prime}=\mathcal{E}\left(q^{\prime}\right)\right]$.

However, the erosion $\mathcal{E}$ of the above theorem may not be the adjoint of the dilation $\Delta$. To form an adjunction, we first define a signal fuzzy dilation as the previous sup- $T$ convolution

$$
\delta(f)(x) \triangleq \bigvee_{y \in \mathbb{E}} T[f(y), h(x-y)]=\left(f \bigcirc_{T} h\right)(x)
$$

By recognizing $T[f(y), h(x-y)]$ as the scalar dilations $\delta_{x, y}(f(y))$ in the general decomposition (1) of $\delta$, it follows that the adjoint signal fuzzy erosion is

$$
\varepsilon(g)(y) \triangleq \bigwedge_{x \in \mathbb{E}} \Omega[g(x), h(x-y)]
$$

where $\Omega$ represents the adjoint scalar erosions $\left(\varepsilon_{y, x}\right)$ in (1) and is actually the adjoint of the fuzzy Tnorm:

$$
T(v, a) \leq w \Longleftrightarrow v \leq \Omega(w, a)
$$

Given $T$ we can find its adjoint function $\Omega$ by

$$
\Omega(w, a) \triangleq \sup \{v \in[0,1]: T(v, a) \leq w\}
$$

The norm $T$ can be interpreted as a logical conjunction, whereas its corresponding adjoint can be interpreted as a logical implication [6].

Three examples of Tnorms are:

$$
\begin{gathered}
\text { Min : } T_{1}(v, a)=\min (v, a), \quad \text { Product }: T_{2}(v, a)=v \cdot a \\
\text { Yager }: T_{3}(v, a)=1-\left(1 \wedge\left[(1-v)^{p}+(1-a)^{p}\right]^{1 / p}\right), p>0 .
\end{gathered}
$$

Proc. 10th International Fuzzy Systems Association World Congress (IFSA03), Istanbul, Turkey, July 2003. Lecture Notes in Computer Science 2715, Springer 2003, pp.412-419. 
The corresponding three adjoint functions are:

$$
\begin{gathered}
\Omega_{1}(w, a)=\left\{\begin{array}{l}
w, w<a \\
1, w \geq a
\end{array}, \quad \Omega_{2}(w, a)= \begin{cases}\min (w / a, 1), & a>0 \\
1, & a=0\end{cases} \right. \\
\Omega_{3}(w, a)= \begin{cases}1-\left[(1-w)^{p}-(1-a)^{p}\right]^{1 / p}, & w<a \\
1, & w \geq a\end{cases}
\end{gathered}
$$

The generalized fuzzy opening $\alpha$ and fuzzy closing $\beta$ are

$$
\alpha(f) \triangleq \delta(\varepsilon(f)), \quad \beta(f) \triangleq \varepsilon(\delta(f))
$$

If we define an alternative erosion operator (as an inf- $U$ convolution) by

$$
\varepsilon^{\prime}(f)(y)=\bigwedge_{x} U[f(x), h(y-x)]
$$

where $U(a, b)=1-T(1-a, 1-b)$ is a fuzzy union that is dual to $T$, then $\varepsilon^{\prime}(f)=1-\delta(1-f)$; i.e., this second erosion $\varepsilon^{\prime}$ is the dual of the first dilation $\delta$. Further, the adjoint dilation $\delta^{\prime}$ of $\varepsilon^{\prime}$ is an operator that is dual of the first erosion $\varepsilon$. Many previous works used pairs $\left(\varepsilon^{\prime}, \delta\right)$ which are duality pairs but not adjunctions and hence cannot form openings/closings via compositions.

\section{Morphological, Fuzzy and Hybrid Edge Gradients}

In this section we present some theoretical and experimental results on using the lattice fuzzy operators, in combination with morphological systems or by themselves, to develop some new edge gradients based on fuzzy operators.

Morphological Gradient: In the continuous case (images defined on $\mathbb{R}^{2}$ ), if $\delta_{s}(f)=f \oplus s B$ and $\varepsilon_{s}(f)=f \ominus s B$ are the flat dilation and erosion of $f$ by multiscale disks $s B$, it is well-known that

$$
\lim _{s \downarrow 0}\left[\delta_{s}(f)(x)-\varepsilon_{s}(f)(x)\right] /(2 s)=\|\nabla f(x)\|
$$

for a differentiable function $f$.

Hybrid Gradient: We have proven that a similar result holds if we replace in (9) the morphological dilation with a multiscale fuzzy dilation as follows:

$$
\delta_{s}(f)(x)=\bigvee_{y} T(f(x-y), h(y / s))
$$

Specifically, assuming that $f$ and $T$ are differentiable, we have proven that

$$
\lim _{s \downarrow 0}\left[\delta_{s}(f)(x)-f(x)\right] / s=K \cdot\|\nabla f(x)\|, \quad K=\bigvee_{y} \partial_{1} T[f(x), h(y)] \cdot y
$$

where $\partial_{1}$ denotes partial derivative w.r.t. first argument. Further, for the fuzzy edge gradients, the unit-scale structuring function $h(x)$ must be a unimodal 
symmetric structuring function with a global maximum at $x=0, h(0)=1$. Similarly we can define an adjoint multiscale fuzzy erosion $\varepsilon_{s}$ and a corresponding symmetric fuzzy gradient $\lim _{s \downarrow 0}\left[\delta_{s}(f)(x)-\varepsilon_{s}(f)(x)\right] /(2 s)$.

Discrete versions of the above gradients results by replacing the above scalenormalized limits with differences $\delta(f)-\varepsilon(f)$, where $\delta$ and $\varepsilon$ represent: i) (in the classic morphological case) a morphological dilation and erosion of $f$ by a $3 \times 3$-pixel flat structuring element, or ii) (in the hybrid case) a fuzzy dilation and erosion by a small structuring element (at scale $s=1$ ). The hybrid (morphological-fuzzy) gradient showed a small improvement over the morphological one.

Fuzzy Gradient: We also propose a new and different type of discrete edge gradient:

$$
\text { FuzzyEdge }_{\min }(f)=\min \left[\delta_{s}(f), 1-\varepsilon_{s}(f)\right]
$$

where $\delta_{s}$ and $\varepsilon_{s}$ are the same fuzzy dilation and erosion as above with $s=1$. A dual type of fuzzy edge gradient, FuzzyEdge ${ }_{\max }$, results when the min is replaced with max. The last two types of edge gradients were inspired by the standard discrete morphological gradient $\delta(f)-\varepsilon(f)$, but to make the gradient operator more consistent with fuzzy set theory we replaced the difference between dilation and erosion with min (or max) of the dilation and the fuzzy complement of the erosion.

In Fig. 1 we present some experimental results illustrating the differences between the classical morphological operators and the generalized lattice-fuzzy operators. Rows 1 and 2 show the dilation, erosion, opening and closing of 1D images. In general, we have observed that, the fuzzy operators are more adaptive and track closer the peaks/valleys of the signal than the corresponding flat morphological operators of the same scale. Similar conclusions were reached during our experiments with 2D images. In Rows 3 and 4, we investigate the performance of the fuzzy operators in edge enhancement-detection. As shown in Fig. 1(h,i), the new fuzzy gradient operators have a quite promising behavior since they yied clean and sharper edges than the morphological gradient. Further, the fuzzy operators are less influenced by noise than the morphological operators. The morphological operators seem to detect more edges in non-noisy images (Fig. 1(n,i)); however, the fuzzy operators may also be designed to detect more edges by optimally tuning the parameter of the Tnorm [9]. The last three rows of Fig. 1 clearly illustrate the superior performance of the fuzzy operators for edge detection in noise. The results are demonstrated by using a test 1D signal with known derivative and edge location. The noisy signal versions are formed by adding 'gray salt and pepper' noise at different SNRs; see Figs. 1(m,n,o). In both the morphological and fuzzy case, the performance, in the presence of noise, is improved with larger size structuring elements.

\section{Conclusions}

The power but also the difficulty in applying the lattice-based fuzzy operators to image analysis is the huge variety of fuzzy norms and the absence of some

Proc. 10th International Fuzzy Systems Association World Congress (IFSA03), Istanbul, Turkey, July 2003. Lecture Notes in Computer Science 2715, Springer 2003, pp.412-419. 
practical experience or systematic ways in selecting them. In general, having a parameter in the Tnorm offers flexibility for the fuzzy operators that use it. The scale (support size) and the shape of the structuring function are also important factors, which can influence the behavior of the fuzzy operators. By tuning these parameters we can find the optimum solution depending on the type of application, such as edge detection in the presence of noise or other feature detection in images with low contrast [9]. In our on-going work we are investigating various methods to design these new operators for various nonlinear filtering and image analysis tasks. Finally, by combining lattice MM and fuzzy set theory, we can create new operators, like the fuzzy edge gradients, that extend and improve the capabilities of the standard morphological operators.

\section{References}

1. I. Bloch and H. Maitre, "Fuzzy mathematical morphologies: a comparative study" Pattern Recognition 28, vol. 9, pp. 1341-1387, 1995.

2. T. Q. Deng and H.J.A.M. Heijmans, "Grey-Scale Morphology Based on Fuzzy Logic", J. Math. Imaging and Vision, 16: 155-171, 2002.

3. V. Goetcherian, "From binary to greytone image processing using fuzzy logic concepts", Pattern Recognition 12, pp.7-15, 1980.

4. H. J. A. M. Heijmans, Morphological Image Operators, Academic Press, Boston, 1994.

5. E. E. Kerre and M. Nachtegael, "Fuzzy Techniques in Image Processing: Techniques and Applications", in Studies in Fuzziness and Soft Computing, Vol. 52, Physica Verlag, 2000.

6. G. J. Klir and B. Yuan, Fuzzy Sets and Fuzzy Logic: Theory and Applications, Prentice-Hall, 1995.

7. P. Maragos, G. Stamou and S. Tzafestas, "A Lattice Control Model of Fuzzy Dynamical Systems in State-Space", in Mathematical Morphology and Its Application to Image and Signal Processing, J. Goutsias, L. Vincent and D. Bloomberg, Eds, Kluwer Acad. Publ., Boston, 2000, pp. 61-70.

8. P. Maragos and S. Tzafestas, "A Lattice Calculus Unification of Min-Max Control Systems of the Morphological and Fuzzy Type", Proc. Int'l Symp. on Soft Computing in Engineering Applications (SOFTCOM-98), Athens, June 1998.

9. P. Maragos, V. Tzouvaras, and G. Stamou, "Synthesis and Applications of Lattice Image Operators Based On Fuzzy Norms", Proc. Int'l Conf. Image Processing (ICIP-2001), Thessaloniki, Greece, Oct. 2001.

10. M. Nachtegael and E. E. Kerre, "Decomposing and Constructing Fuzzy Morphological Operations Over $\alpha$-Cuts: Continuous and Discrete Case", IEEE Trans. Fuzzy Systems, vol.8, pp.615-626, Oct. 2000.

11. Y. Nakagawa and A. Rosenfeld, "A note on the use of local min and max operations in digital picture processing", IEEE Trans. Syst., Man, Cybern., SMC-8, p.632-635, Aug.1978.

12. J. Serra Ed., Image Analysis and Mathematical Morphology II: Theoretical Advances, Acad. Press, NY, 1988.

13. D. Sinha and E. R. Dougherty, "Fuzzy mathematical morphology", J. Visual Communication and Image Representation, vol. 3, no. 3, pp. 286-302, 1992.

14. L. A. Zadeh, "Fuzzy Sets", Information and Control, vol. 8, pp. 338-353, 1965.

Proc. 10th International Fuzzy Systems Association World Congress (IFSA03), Istanbul, Turkey, July 2003. Lecture Notes in Computer Science 2715, Springer 2003, pp.412-419. 

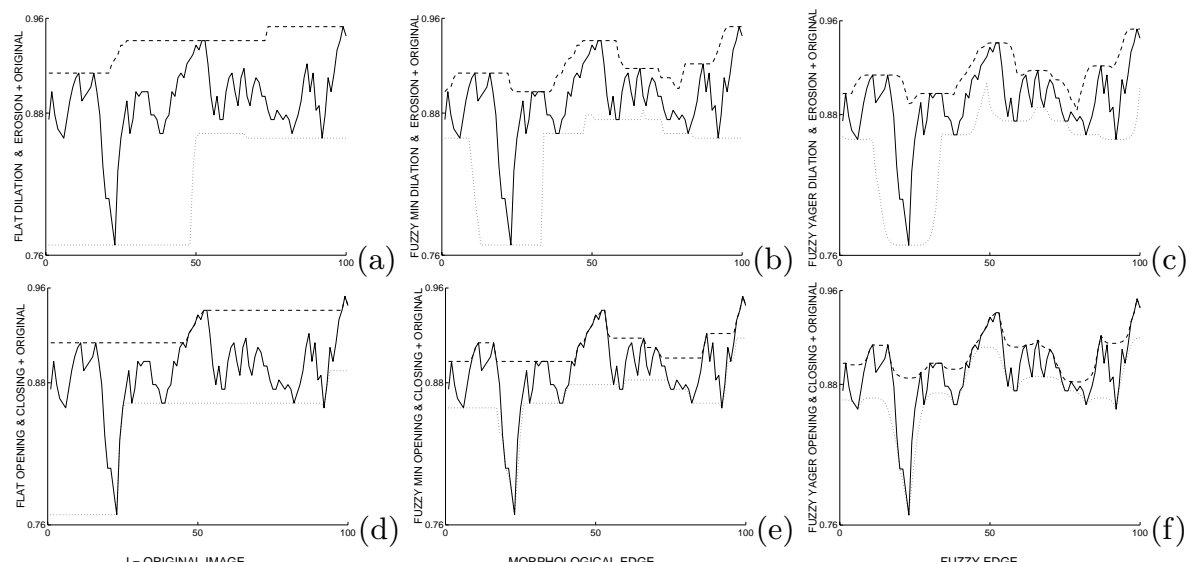

\section{IFSA 2003}
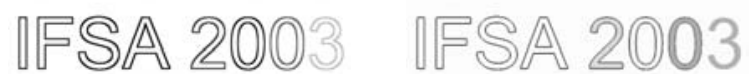

(g)

(h)

(i)
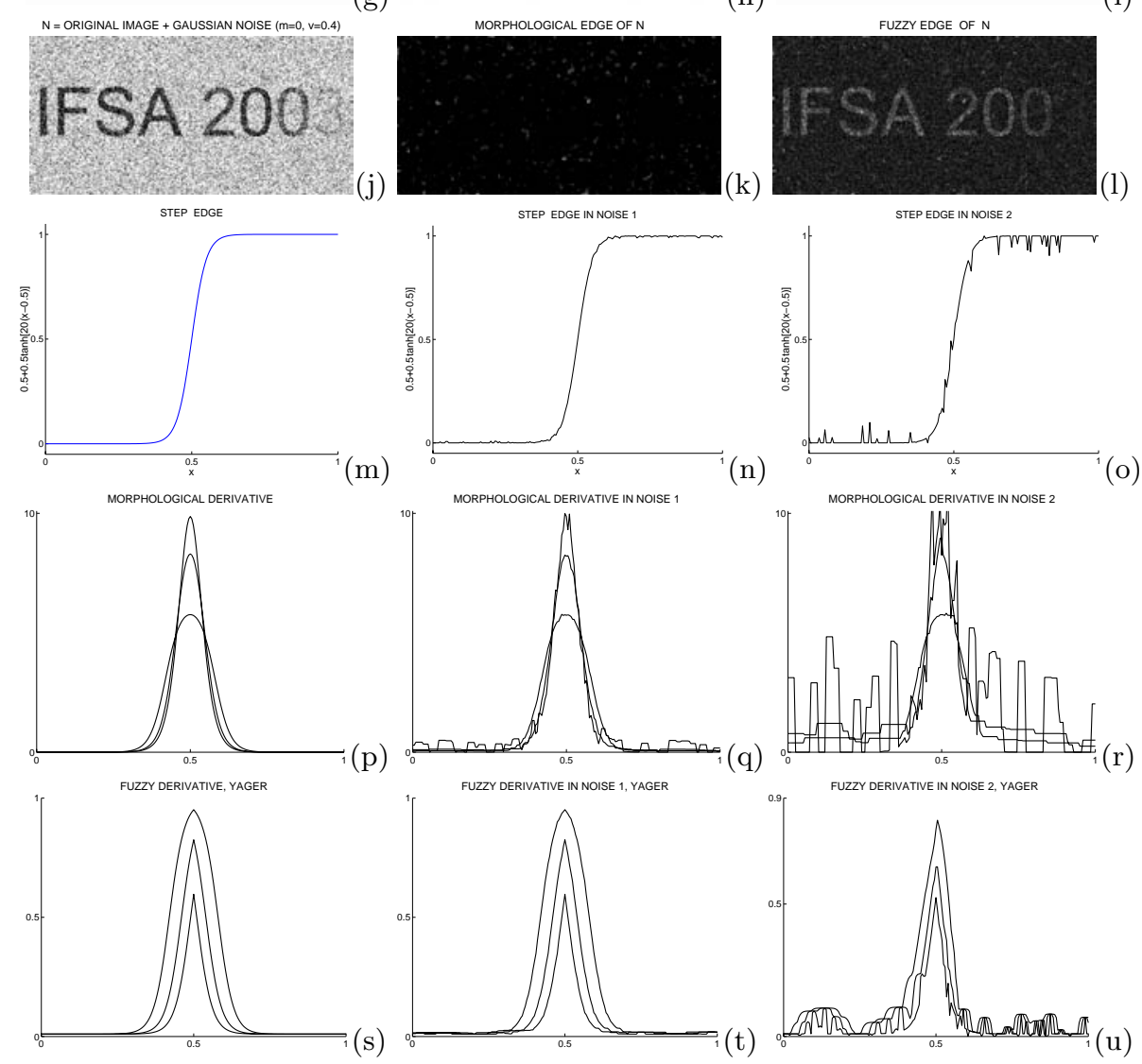

Fig. 1. Rows 1 and 2, left to right: flat, minimum, Yager. Row 1: original signal (solid line), dilation (dashed line), erosion (dotted line). Row 2: closing (dashed line), opening (dotted line). Row 3: Edges in images. Row 4: Edges in noisy images Row 5: step edge function, signal with low noise, signal with high noise. Rows 6,7: performance of morphological and fuzzy edge operators, respectively.

Proc. 10th International Fuzzy Systems Association World Congress (IFSA03), Istanbul, Turkey, July 2003. Lecture Notes in Computer Science 2715, Springer 2003, pp.412-419. 